\title{
Reliability Analysis of the Regasification System on Board of a FSRU Using Bayesian Networks
}

\author{
Marcelo Ramos Martins and Adriana Miralles Schleder
}

Additional information is available at the end of the chapter

http://dx.doi.org/10.5772/45803

\section{Introduction}

The global economic downturn has reduced the demand for all types of energy while the world's capacity to produce natural gas is surging. As a result, LNG is becoming an important energy source option. Large reserves of natural gas exist around the world, often in areas where there is no market or where the resources exceed the demand. Therefore, this natural gas must be shipped to areas where there is demand, and, to reduce costs, the gas is liquefied, reducing its volume by about 600 times. Thus, the storage and regasification system usually occurs in onshore plants. The LNG is stored in a double-walled storage tank at atmospheric pressure until needed. Then, the LNG is pumped at a higher pressure and warmed until it turns into gas again.

From this viewpoint, the FSRU (Floating and Storage Regasification Unit) is becoming a new economic and flexible alternative for the storage and regasification system. The FSRU costs less than an onshore facility of similar capacity; it provides a faster return to the capital invested because time is saved by not having an extensive planning and permitting process as usually occurs with onshore developments. Moreover, construction time is reduced, assuming the conversion of an existing LNG carrier. Additionally, as the FSRU can be moved from one demand area to another, paperit is a flexible and attractive feature in countries with seasonal demand or where there is an unstable market.

It is also worth noting two more FSRUs attractive features. First, an accident in one onshore plant might produce considerable impact on neighboring areas and on the local population (this risk may be even worse due to the possibility of a terrorist attack), as reported by [1, 2]. Second, LNG provides clean energy as compared with traditional fuels, and it is a significant alternative to diversify the national energy matrix.

Because the regasification system usually occurs in onshore plants, the processing in vessels is pioneering. These vessels were formerly used for transporting liquefied gas and were 
transformed into FSRUs to gasify the LNG. Therefore, the development of efficient technologies for LNG exploration and distribution is essential. Risk and reliability analysis are vital to the development of these technologies.

As reported by [3], an increasing number of recent studies have applied BN in reliability analysis, risk analysis and maintenance due to the benefits that $\mathrm{BN}$ provides in contrast with traditional tools, such as its capacity for representing limited or incomplete knowledge, local dependencies and multi-state variables and its ability to deal with any probability density function. Its practicality to model common cause failures and the capability to update the state of knowledge according to new evidence are also important advantages that have been explored in reliability analysis using BN.

This chapter proposes the construction of a $\mathrm{BN}$ to evaluate the reliability of the regasification system of a FSRU and presents additional developments over a previous published study [4], in which the regasification system BN, converted from a fault tree, was evaluated to model the probability of an undesired event. The first part of this chapter provides an overview of the BNs, followed by explanations of the Regasification System as well as the Regasification System BN. The model shown herein was built and executed using the commercial tool, AgenaRisk, available in [5]. In the second part of this chapter, the reliability of the regasification system is evaluated and information is obtained such as critical components and subsystems and other conditions that affect the system reliability.

\section{Bayesians networks}

As defined by [6], $\mathrm{BN}$ is a graphical structure for representing the probabilistic relationships among a large number of variables and for making probabilistic inferences with those variables. A BN is a direct acyclic graph (DAG) with the nodes representing the variables and the arcs, their conditional dependencies. The BN qualitative analysis provides the relationships between the nodes while the quantitative analysis may be performed in two ways: a predictive analysis or a diagnostic analysis. The first one calculates the probability of any node based on its parent nodes and the conditional dependencies. The second one calculates the probability of any set of variables given some evidence.

The nodes and arcs are the qualitative components of the networks and provide a set of conditional independence assumptions that may be represented through a graph notion called d-separation, which means that each arc built from variable $X$ to variable $Y$ is a direct dependence, such as a cause-effect relationship.

If the variables are discrete, the probabilistic relationship of each node $X$ with their respective parents $\mathrm{pa}(\mathrm{X})$ is defined by its Conditional Probability Table $(\mathrm{CPT})$ while for continuous variables, this probabilistic relationship is defined by its Conditional Probability Distribution (CPD), which represents conditional probability density functions. The quantitative analysis is based on the conditional independence assumption. Considering three random variables $X, Y$ and $Z, X$ is said to be conditionally independent of $Y$ given $Z$, if $\mathrm{P}(\mathrm{X}, \mathrm{Y} \mid \mathrm{Z})=\mathrm{P}(\mathrm{X} \mid \mathrm{Z}) \mathrm{P}(\mathrm{Y} \mid \mathrm{Z})$. The joint probability distribution of set of variables, based on their conditional independence, can be factorized as shown in Eq.1: 


$$
\mathrm{P}\left[\mathrm{x}_{1}, \mathrm{x}_{2}, \ldots, \mathrm{x}_{\mathrm{n}}\right]=\prod_{\mathrm{i}=1}^{\mathrm{n}} \mathrm{P}\left[\mathrm{x}_{\mathrm{i}} \mid \text { Parent }_{\mathrm{i}}\right]
$$

The graphical representation is the bridging of the gap between the (high level) conditional independence statements that must be encoded in the model and the (low level) constraints, which enforce the CPD [7].

Given some evidence, beliefs are recalculated to indicate their impact on the network. The possibility of using evidences of the system to reassess the probabilities of network events is another important feature of the BNs; it is interesting to determine critical points in the system. Classical methods of inference of a BN for this purpose involve computation of the posterior marginal probability distribution of each component, computation of the posterior joint probability distribution of subsets of components and computation of the posterior joint probability distribution of the set of all nodes. The analysis and propagation of evidences allowed by BN are useful to explore or forecast some system behavior that is unknown or requires more attention as in [8]. In addition, the propagation of evidences offers the possibility to check the influence of redundant systems or critical equipment, such as equipment that requires a long time to repair.

In the last years, the number of studies that presented the use of $\mathrm{BN}$ in reliability analysis have been increased [9-11]; traditional models, such as fault trees and block diagrams, have been replaced by discrete BN. However, to perform an efficient application of BNs in reliability assessment, such network models must be hybrid models formed by discrete and continuous variables. The evaluation of hybrid networks poses a challenge; there are limitations of inference algorithms, such as dealing with state space explosion and finding an appropriate discretization. However, a new and efficient dynamic discretization of the domain and an iterative approximation method which produce finer discretization in the regions that contribute more to the structure of the density functions associated with a robust propagation algorithm were proposed by $[9,10]$. This approach is implemented in the commercial BN software package, AgenaRisk [5], which is used in this study. There are other commercial tools for the calculation of BNs, such as [12] and [13]. [14] is an excellent reference for the theoretical aspects of $\mathrm{BNs}$ and algorithms. This approach allows the $\mathrm{BN}$ to deal with any probability distribution function, unlike the traditional tools which are capable of dealing only with exponential distributions.

Another relevant $\mathrm{BN}$ feature is its practicality to model common cause failures; the Common Cause Failures (CCFs) are the failure of more than one component due to the same cause, which can render the redundancy protection useless significantly affecting the system reliability. More details about CCFs and proposed methods to deal with are reported in $[16,17]$. Including CCFs in a $\mathrm{BN}$ is not a complex process. If the $\mathrm{BN}$ is a network composed of only discrete variables, the CCF probabilities are included directly to the CPT, as [10] reports, and it is not necessary to use additional constructs such as when using fault trees. If the $\mathrm{BN}$ is a network composed of continuous variables, the CCFs are represented by additional nodes; one node for each group of similar components is included in the BN. The frequency rate of these failures is estimated according to the redundant components; $[16,17]$ explain the methods for obtaining these rates. 
It is also worth noting that $\mathrm{BNs}$ are efficient to model multi-state variables, local dependencies and limited or incomplete knowledge. Multi-state variables and local dependencies are important to build a more realistic model; traditional tools usually use just binary variables and are unable to represent local dependencies, for example to represent how a malfunction of the equipment affects other equipment. Occasionally, there are not enough or satisfactory statistical data about the system to perform the reliability analysis; in this situation, $\mathrm{BN}$ builders ask relevant questions to a group of specialists and explain the assumptions that are encoded in the model, and the domain experts supply their knowledge to the BN builders ([18] and [19] demonstrated this process).

The reliability analysis presented in this chapter explores the benefits of BN use, such as the inclusion of CCFs, modeling with continuous variables, propagation of evidence and local dependences representation.

\section{Methodology}

The proposed methodology is a combination of different techniques already used. Proposals of different authors and several techniques were combined to compose the methodology, which resulted in the formation of a four-step methodology: familiarization, qualitative analysis, quantitative analysis and complementary analysis.

In the first step, familiarization, all the information available about the system and the operation must be collected. The second step, qualitative analysis, is the step at which the relationship among the system components must be identified and, as a result, a BN is built to represent the system. Next, in the quantitative analysis, the priori probabilities of root nodes and the conditional probabilities tables for non-root nodes are defined allowing the evaluation of the joint probability of a set of variables. Finally, the complementary analyses must be performed by evaluating the posterior probabilities: criticality analysis, the analysis of different scenarios of interest and the conditional reliability analysis. These analyses allow improving the reliability analysis through an evaluation that is not possible through traditional tools. The criticality analysis means to find the set of components or subsystems that have greater influence in the system behavior; the analysis of different scenarios can be used to model any situation of interest, such as the impact of including redundancies, the impact of a component fault or any other condition that affects the system reliability; and the conditional reliability analysis provides information about the system behavior over time. Figure 1 presents an overview of the methodology steps; the figure is divided into two parts: the first in which all the tasks to be performed at each step are listed and the second which lists the means suggested for these tasks.

\section{Application}

In this section, the reliability analysis of the regasification system is performed by using the methodology of the previous section. First, the information collected about the system is presented. Then, the qualitative analysis is performed. Subsequently, a quantitative analysis 


\begin{tabular}{|c|c|c|c|c|}
\hline & $\begin{array}{c}\text { Step } 1 \\
\text { Familiarization }\end{array}$ & $\begin{array}{c}\text { Step } 2 \\
\text { Qualitative Analysis }\end{array}$ & $\begin{array}{c}\text { Step } 3 \\
\text { Quantitative Analysis }\end{array}$ & $\begin{array}{c}\text { Step } 4 \\
\text { Complementary Analyses }\end{array}$ \\
\hline$\frac{n}{n}$ & $\begin{array}{l}\text { Understanding the } \\
\text { system and } \\
\text { identifying possible } \\
\text { scenarios that the } \\
\text { system will be } \\
\text { submitted to. }\end{array}$ & $\begin{array}{l}\text { Representing the } \\
\text { system physically and } \\
\text { functionally; } \\
\text { Representing the } \\
\text { relationships between } \\
\text { system elements; }\end{array}$ & $\begin{array}{l}\text { Completing the } \\
\text { construction of BN with } \\
\text { quantitative data (priori } \\
\text { probabilities, CPT and } \\
\text { density functions); } \\
\text { Estimating the } \\
\text { probability density of } \\
\text { system failure and } \\
\text { reliability for a given } \\
\text { mission time. }\end{array}$ & $\begin{array}{l}\text { Analyzing the criticality; } \\
\text { Analyzing different } \\
\text { scenarios; } \\
\text { Analyzing the conditional } \\
\text { reliability. }\end{array}$ \\
\hline 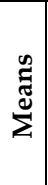 & $\begin{array}{l}\text { Data review; } \\
\text { Interviews with } \\
\text { experts. }\end{array}$ & $\begin{array}{c}\text { Functional tree; } \\
\text { Unifilar diagram; } \\
\text { Block diagram; } \\
\text { Fault tree; } \\
\text { Bayesian Networks. }\end{array}$ & $\begin{array}{l}\text { Bayesian Network } \\
\text { (Inference) }\end{array}$ & $\begin{array}{l}\text { Bayesian Network } \\
\text { (posterior probability) }\end{array}$ \\
\hline
\end{tabular}

Figure 1. Methodology

will be conducted, in which the failure probability density and the system reliability are estimated for a given mission time of the system. And finally, the complementary analysis is presented. The AgenaRisk (Desktop Agena Risk, 2011)[5] was used to build the Bayesian Network and to make the inferences about the system.

\subsection{Familiarization - The regasification system}

Usually, vessels are used for LNG transportation; however, in the last years, these vessels also began to participate in gas regasification and directly supply net pipes. The regasification process onboard adds new hazards to the operations of LNG vessels, because in addition to LNG, there is now compressed gas in the process. Accidents in this process may reach the storage tanks, causing very severe consequences.

In the vessel studied, a cascade system was used to regasify the LNG. In this system, the LNG is heated in two stages. In the first, it is heated by a propane compact heat exchanger (HE1), and its temperature increases from $-162^{\circ} \mathrm{C}$ to $-10^{\circ} \mathrm{C}$; at this stage, the natural gas is already vaporized, but this temperature is too low for delivery to the pipeline, where the heating process would continue. In the next stage, the gas is heated by seawater in a shell-and-tube heat exchanger (HE2), and the temperature reaches $15^{\circ} \mathrm{C}$. The first stage uses no water due to the possibility of water freezing in direct contact with LNG. The propane used in the first phase works in a closed loop. When the propane leaves the LNG heat exchanger HE1, its temperature is approximately $-5^{\circ} \mathrm{C}$, and it is liquefied (propane at 4.7 bar liquefies at approximately $-5^{\circ} \mathrm{C}$ ); hence, it is pumped into a titanium heat exchanger (HE3) and heated, by sea water, up to $0^{\circ} \mathrm{C}$ at 4.7 bar and vaporizes. It then returns to the LNG exchanger HE1. This system must have an effective thermal insulation to avoid an unexpected heat gain of the LNG or propane inside the tubing, which could result in a gas expansion and possibly cause a tubing rupture. A more detailed description of the regasification system is given in [4]. A 
diagram of the system is shown in Figure 1, and the nomenclature used is presented in Table 1. The thermal insulation for each heat exchanger will also be considered (I1, I2 and I3).

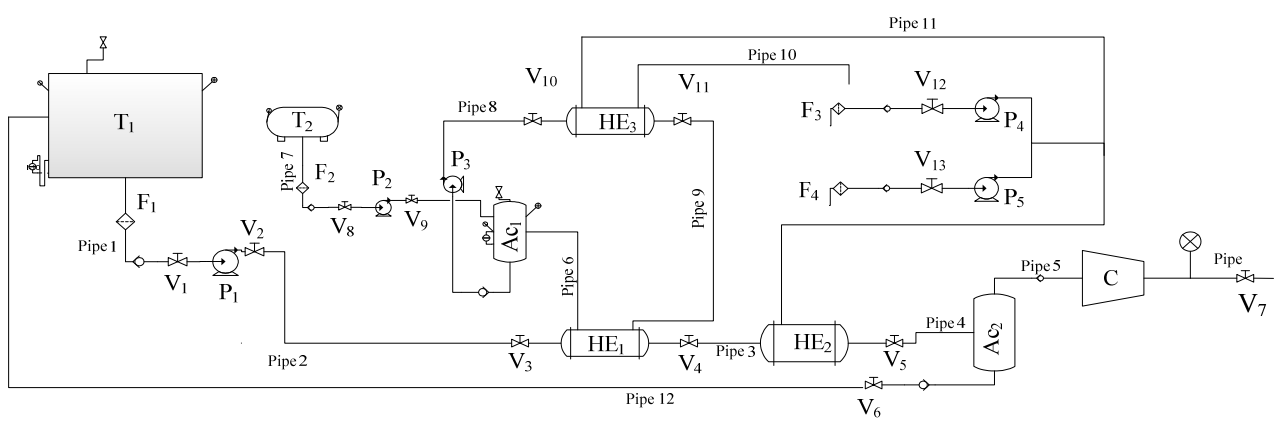

Figure 2. Regasification system

\begin{tabular}{|cccc|}
\hline Item & Description & Item & Description \\
\hline T1 & LNG tank & P2, P3 & Propane pumps \\
T2 & Propane tank & P4, P5 & Water pumps \\
V1, V2, V3, V6, V8, V9, & Gate valves for liquid & $\begin{array}{c}\text { Pipes 1, 2, 3, 4, 5, 6, 7, } \\
\mathbf{8 , 9 , 1 0 , 1 1 , 1 2}\end{array}$ & Pipes with less than 10" \\
V10, V11, V12, V13 & Gate valves for gas & Delivery Pipe & Pipe with 12" \\
V4, V5, V7 & LNG supply pump & Ac1 & $\begin{array}{c}\text { Propane accumulator } \\
\text { Propane filter }\end{array}$ \\
P1 & LNG filter & F2 & Sea water/LNG shell\&tube \\
F1 & LNG accumulator & HE2 & heat exchanger \\
Ac2 & Propane/LNG compact & HE3 & Propane/sea water \\
HE1 & heat exchanger & titanium heat exchanger \\
C & Compressor & F3 e F4 & Water filter \\
\hline
\end{tabular}

Table 1. Nomenclatures

\subsection{Qualitative analysis}

Qualitative analysis should provide a clear view of the system and the relationships between system elements; this representation may be produced by building a block diagram or a fault tree and then converting it into $\mathrm{BN}$, as presented by [4] and [11] or may be directly produced from the system analysis.

The regasification system was represented in a hybrid BN. Continuous nodes were built to represent the time to failure (TTF) of the basic components and subsystems, and discrete nodes to represent the state of the system or subsystem.

Another important BN feature is its capability of modeling local dependences. The regasification system has local dependences between heat exchangers and insulators; the failure probability distributions of the nodes "Heat Exchanger LNG/Propane" (HE1), "Heat Exchanger LNG/Water" (HE2) and "Heat Exchanger Propane/Water" (HE3) change if the insulation fails. If the insulation fails, the heat exchanger failure probability increases. This 
variable has a conditional dependence that is not possible to address with traditional approaches such as Fault Trees (FT). In FT analysis, it is not possible represent local dependence, but it can be modeled in a simple way with BNs. To include this dependence in the model, an arc was built between these nodes (Figure 3). With this approach, it is possible to model how the malfunction of any equipment affects other equipment.

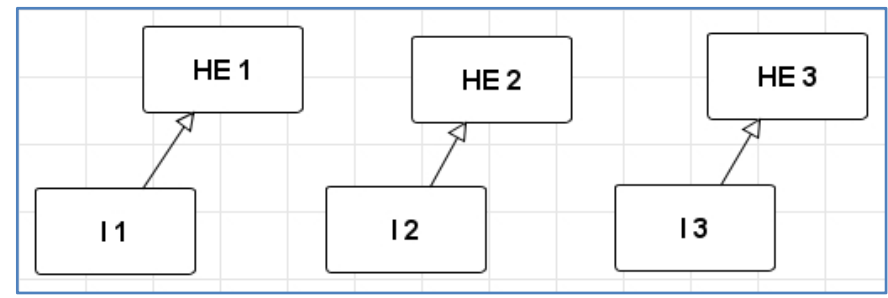

Figure 3. Conditional dependences

Finally, to complete the qualitative analysis, the CCF must be included. This system has a redundant subsystem in which a CCF may occur; a parallel system provides water for heat exchangers HE2 and HE3. The CCFs are important contributors to system unreliability and typically exist among redundant units. A CCF in this subsystem directly affects the reliability of the whole system. In the $\mathrm{BN}$, one node is included for each group of redundant components to verify the CCF effects; each node is a representation of the CCF associated with groups of similar equipment: CCF1 (valves), CCF2 (filters) and CCF3 (pumps).

The Regasification System BN is illustrated in Figure 4; the top node represents the whole regasification system. Below are the subsystems and even the basic components and the CCFs are highlighted. Also, there is node " $R$ " which is the reliability node; it will be used to evaluate the system reliability for a specific mission time.

\subsection{Quantitative analysis}

Quantitative analysis begins with the inclusion in the $\mathrm{BN}$ of the priori probabilities of root nodes; these probabilities can be provided by statistical data or be estimated by experts. Next, the relationships between nodes must be specified. And finally the joint probability of the network is obtained, which, in the case study will serve to obtain the system reliability for a given mission time. The root nodes that represent the basic components are completed by probability density functions representing the TTF of each basic component.

The relationships between components are represented by basic constructs, such as the AND and OR gates, used in fault trees. The AND gate, where the output will fail when all input components fail, has a probability of failure of its output in the time interval $[0, t]$, given by:

$$
\mathrm{P}\left(\zeta_{\text {And }} \leq \mathrm{t}\right)=\mathrm{P}\left(\zeta_{1} \leq \mathrm{t}, \ldots, \zeta_{\mathrm{n}} \leq \mathrm{t}\right)=\mathrm{P}\left(\max \left\{\zeta_{\mathrm{i}}\right\} \leq \mathrm{t}\right)
$$

Where $\zeta_{\text {And: }}$ time to failure of AND gate

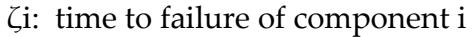




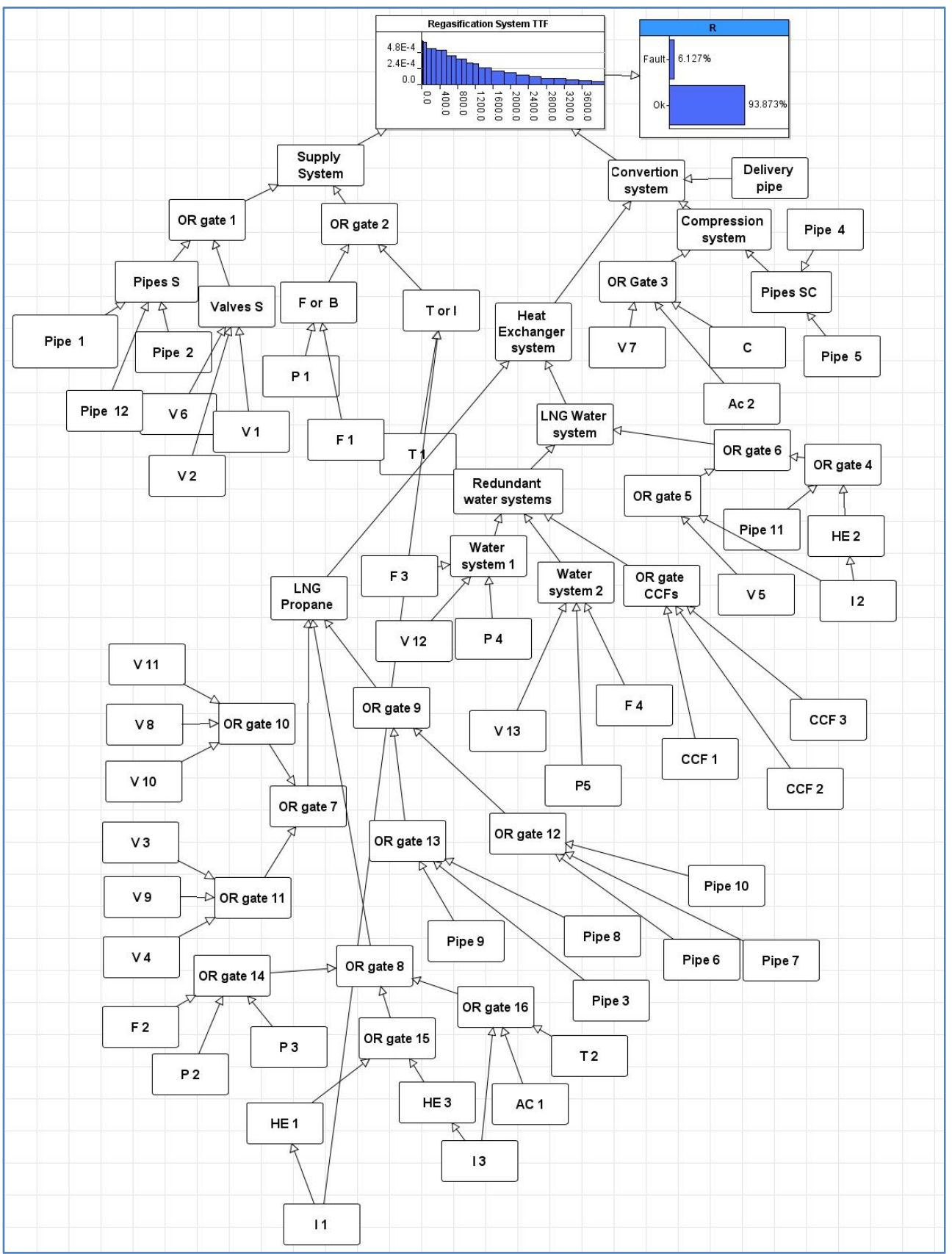

Figure 4. Regasification system BN 
The OR gate, where the output will fail if at least one input component fails, has a probability of failure of its output, in the time interval $[0, t]$, given by:

$$
\left.\mathrm{P}\left(\zeta_{\mathrm{OR}} \leq \mathrm{t}\right)=1-\mathrm{P}\left(\zeta_{1}>t, \ldots, \zeta_{\mathrm{n}}>t\right)=\mathrm{P}\left(\min \left\{\zeta_{\mathrm{i}}\right\} \leq \mathrm{t}\right)\right)
$$

where

$$
\begin{aligned}
& \zeta_{\text {OR: time to failure of OR gate }} \\
& \zeta_{\text {i: }} \text { time to failure of component } i
\end{aligned}
$$

Although $\mathrm{BN}$ is able to deal with any kind of prior distribution, the components were considered to have constant failure rates $(\lambda)$ which means that the time-to-failure distributions were assumed to be exponential. Thus, the probability of a component to fail at time $\mathrm{T}$ within a given mission time $\mathrm{t}$ is calculated as $\mathrm{P}(\mathrm{T}<\mathrm{t}))=1-\mathrm{e}^{-\lambda t}$, except for the insulation. Statistical data about the probability of the insulation failure were not found along this investigation, but these distributions may be estimated by expert judgment. BN builders ask relevant questions to a group of specialists and explain the assumptions that are encoded in the model, and the domain experts supply their knowledge to the BN builders. [18,19] demonstrated this process. In the current study, for the insulator node, the failure distribution was assumed to be a Weibull distribution with shape factor $s=6$ and inverse scale $\beta=1 / 10000$. It is worth noting that any distribution can be used in the $B N$, which is

\begin{tabular}{|c|c|c|c|}
\hline Component & $\begin{array}{l}\text { Failure } \\
(\lambda) \\
\left(\mathbf{h r}^{-1}\right) \\
\end{array}$ & Component & $\begin{array}{ll}\text { Failure rate } \\
(\lambda) \\
\left(\mathbf{h r}^{-1}\right) & \\
\end{array}$ \\
\hline C & $1.709 \times 10^{-4}$ & $\mathrm{~T} 2$ & $2.883 \times 10^{-5}$ \\
\hline P1 & $4.801 \times 10^{-5}$ & Ac1 & $2.883 \times 10^{-5}$ \\
\hline P2, P3 & $4.801 \times 10^{-5}$ & Ac2 & $2.883 \times 10^{-5}$ \\
\hline HE1 & $3.857 \times 10^{-5}$ & P4, P5 & $5.120 \times 10^{-6}$ \\
\hline HE3 & $2.175 \times 10^{-5}$ & $\begin{array}{l}\text { V1, V2, V3, V6, V8, V9, V10, V11, V12, } \\
\text { V13 }\end{array}$ & $6.610 \times 10^{-6}$ \\
\hline $\begin{array}{l}\text { Pipes } 1,2,3,4,5,6,7,8,9,10,11 \text { ， } \\
12\end{array}$ & $6.700 \times 10^{-9}$ & HE2 & $2.739 \times 10^{-5}$ \\
\hline Delivery pipe & $6.300 \times 10^{-9}$ & $\mathrm{~T} 1$ & $2.523 \times 10^{-5}$ \\
\hline V4, V5, V7 & $1.517 \times 10^{-5}$ & Filters $1,2,3,4$ & $4.155 \times 10^{-7}$ \\
\hline
\end{tabular}
another benefit that BNs offer over those of traditional tools. The failure rates (provided by [20] and [21] are listed in Table 2).

Table 2. Failure rates

As mentioned in the previous section, there are local dependencies between the heat exchangers and insulation. In order to model these dependencies, the failure rates of the heat exchangers were adjusted: the first considers the failure rate of the exchanger in the case the insulation works and the second considers a failure rate higher for the heat exchanger if the insulation fails. Thus, for heat exchanger HE1, the failure rate increases from $3,857 \times 10^{-5}$ to $4,000 \times 10^{-5}$ when the insulation fails, and similarly for the other two heat exchangers, these failure rates are in Table 3. 


\begin{tabular}{|c|c|}
\hline State of insulation & $\begin{array}{c}\text { Failure rate }(\boldsymbol{\lambda}) \\
\left(\mathbf{h r}^{-1} \mathbf{)}\right.\end{array}$ \\
\hline $\mathbf{I}_{1}$ & $\mathrm{HE}_{1}$ \\
Ok & $3,857 \times 10^{-5}$ \\
Fault & $4,000 \times 10^{-5}$ \\
& \\
$\mathbf{I}_{2}$ & $\mathrm{HE}_{2}$ \\
Ok & $2,739 \times 10^{-5}$ \\
Fault & $3,700 \times 10^{-5}$ \\
& \\
$\mathbf{I}_{3}$ & $\mathbf{H E} 3$ \\
Ok & $2,175 \times 10^{-5}$ \\
Fault & $3,000 \times 10^{-5}$ \\
\hline
\end{tabular}

Table 3. Conditional dependences

Finally, to complete, the BN are included the CCF frequency rate; this was calculated using the Beta Factor Model, which was presented by [16] , and the factor beta was assumed to be $\beta=0.1$, as recommended by [17]. These frequency rates are in Table 4 .

The use of BN allowed the inclusion of CCFs in the model despite the use of continuous variables, which is not possible with traditional tools such as fault trees and diagram blocks.

\begin{tabular}{|lcc|}
\hline & CCF Groups & $\begin{array}{c}\lambda \text { group } \\
\lambda_{g}=\boldsymbol{\beta} \boldsymbol{\lambda}_{\boldsymbol{c}}\end{array}$ \\
\hline $\mathbf{1}$ & Valves & $6.610 \times 10^{-7}$ \\
$\mathbf{2}$ & Water pumps & $5.120 \times 10^{-7}$ \\
$\mathbf{3}$ & Water filters & $0.416 \times 10^{-7}$ \\
\hline
\end{tabular}

Table 4. CCF Groups

The $\mathrm{BN}$ was evaluated at a mission time $\mathrm{t}=96$ hours, which is the time required to regasify all of the stored gas in the vessel. The inference of this BN, with all parameters, allows obtaining the prior probability for node $\mathrm{R}$ (which represents the Regasification System Reliability at a mission time), and, for a mission time $96 \mathrm{~h}, \mathrm{R}=0.93873$. The prior reliability system is the first information provided by the $\mathrm{BN}$; however, the $\mathrm{BN}$ may provide many more data, and it allows several analyses concerning the system behavior.

\subsection{Complementary analyses}

\subsubsection{Criticality analysis}

The criticality analysis allows verifying which component or subsystem causes the most impact at the reliability system by evaluating the posterior probabilities. In this chapter, the first criticality analysis performed concerned the impact of each subsystem on the system reliability.

The graph in Figure 5 is a visual perspective, where the length of the bars is a measure of the impact of each node on the target node (the TTF of the Regasification System). The first bar 
indicates the range between the lowest and the highest value for the expected TTF to the regasification system given the LNG/Propane subsystem. The initial point of the bar (equal to 0.5 ) is the expected value for the regasification system TTF given the LNG/Propane subsystem's TTF being nearly zero. The end point (equal to 2569) is the expected value of the regasification system TTF given the LNG/Propane subsystem's TTF significantly exceeds the mission time, which influences the regasification system TTF. After this point, the regasification system TTF stabilizes. Even if the LNG/Propane subsystem's TTF increases, the regasification system TTF does not change. The second bar represents the expected values for the regasification system's TTF conditioned on the Compression subsystem's TTF. The Compression subsystem TTF begins to influence the regasification system TTF when the expected TTF of the regasification system is around 0.5 and this influence ends at the point that the expected regasification system's TTF is 2427 . The next bars are plotted using the same concept.

The graph shows that the most critical subsystem is the LNG/Propane subsystem, which has the most significant influence on the regasification system TTF, and, as expected, the subsystems that have minor influence on reliability are those that are redundant: the water subsystem.

This analysis may also be performed to find critical components; it is an effective tool to search critical points in the system, which contributes to map equipment and subsystems that require improvement and special attention in the maintenance plan. Once seen that the critical subsystem is the LNG/Propane subsystem, the criticality analysis of this subsystem can be performed; this analysis shows that the critical components of the LNG/Propane subsystem are pumps P2 and P3.

In this study, a diagnosis analysis was also performed: given the evidence that the regasification system failed, which component is likely to cause the fault. The failure evidence is included in BN and the beliefs are recalculated. The graph in Figure 6 is the visual perspective where the length of the bars is a measure of the impact of each component on the Regasification system TTF given the evidence "fault of the system". As can be seen, the compressor is the component that has the most influence on the Regasification System TTF given the fault system.

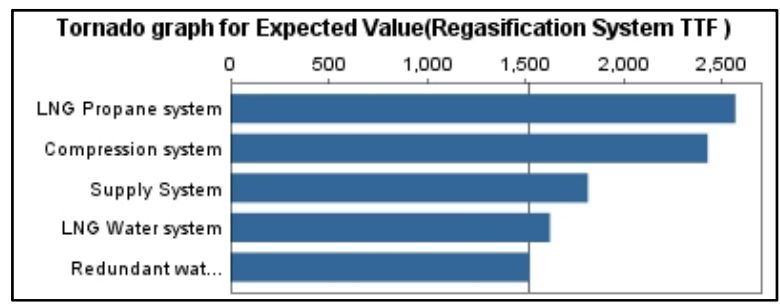

Figure 5. Criticality of the subsystems 


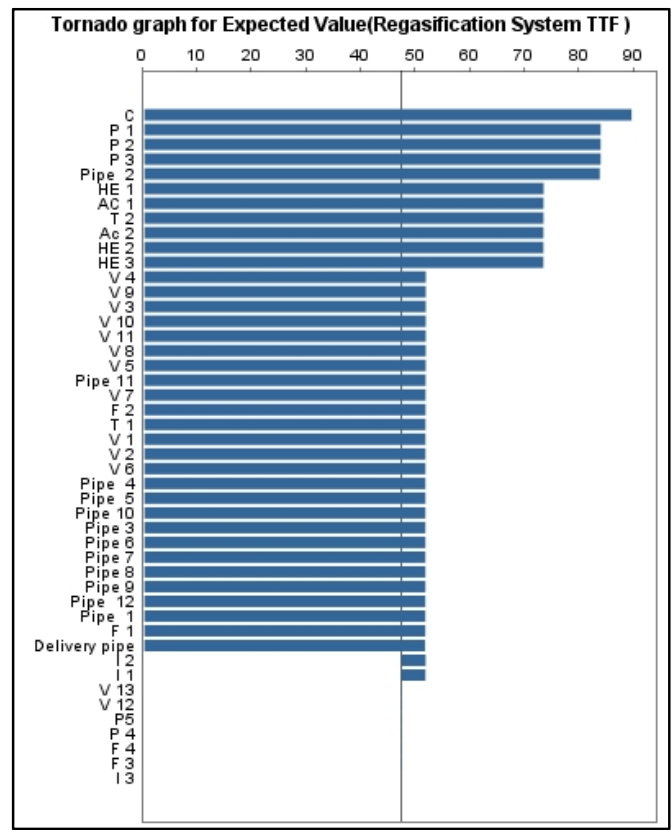

Figure 6. Impact of each component on the Regasification system TTF given the fault system

\subsubsection{Analysis under different scenarios}

$\mathrm{BN}$ allows the study of the evidence propagation and this way the study of different scenarios by evaluating the posterior marginal probability distribution.

Assuming that there is interest in improving the reliability of the regasification system, it is reasonable to consider improving the reliability of the most critical subsystem, but there is generally an associated cost. Thus, the $\mathrm{BN}$ can be used to verify the change in the system reliability due to the improvement of the critical component or subsystem. It is only necessary to include the evidence in the model to perform this analysis. In other words, a scenario is created by assuming that the LNG/Propane subsystem does not fail during the mission time, and then the beliefs are recalculated given this evidence. Thus, it is possible to see the reliability of the system improve from $R=0.93873$ to $R=0.96143$. This is the greatest possible improvement in the system reliability acting on this subsystem (LNG/Propane subsystem). As mentioned in item 4.4.1, the critical components of this subsystem are pumps P2 and P3; thus, a scenario is created in which the evidence that these two pumps will not fail at mission time is included; the beliefs are recalculated to verify the improvement that can be reached by improvements in the reliability pumps. The greatest possible improvement in the system reliability acting on pumps P2 and P3 made the reliability system increase from 0.93873 to 0.94735 .

Another issue is to improve the system reliability by adding a redundancy; in our case, for example, a redundant compressor, once this is the critical component given the fault system. 
However, this action also incurs costs; thus, it is necessary to know the real effect of adding this redundancy. $\mathrm{A} B N$ is an effective way to model the appropriateness of adding a redundancy. A new node that represents the redundant compressor was included in the BN, and then the beliefs were recalculated to indicate the impact on the system reliability. For the system presented here, the reliability increased from $R=0.93873$ to $R=0.95721$. The same analysis may be performed to calculate the effects of a redundant compression subsystem in addition to a redundant component.

The evaluation of the real effects of a redundancy inclusion on the system is essential in decision making process about resources and tasks to improve the reliability system. A BN allows performing this analysis using a simple method.

\subsubsection{Conditional reliability}

The failure rates of the equipment with exponential failure distributions are constant; however, the failure rates of the equipment that have a different distribution modify over time; subsequently, the reliability is modified. The BN allows including a different previous operation time for each component by the inclusion of a node that indicates if a components has a previous operation time. Then, the beliefs are recalculated and the posterior probabilities are evaluated given this evidence.

As an application, a previous operation time of 1000 hours was included as evidence to the insulators, which have a Weibull distribution. Figure 5 shows a partial view of the system BN, in which the extra node which indicates if there is a previous operation time can be seen. In blue, the posterior probabilities given a previous operation time are illustrated, and in green, a new component is illustrated, without a previous operation time. It is possible to simulate different previous operation times for each equipment. For a mission time $96 \mathrm{~h}$, the failure probability of the insulator increases from $9.499 \times 10^{-13}$ to $5.094 \times 10^{-5}$ and the reliability system decreases from 0.93873 to 0.93859 . This analysis allows checking the conditional reliability for any component operation time and, therefore, to know the reliability over time.

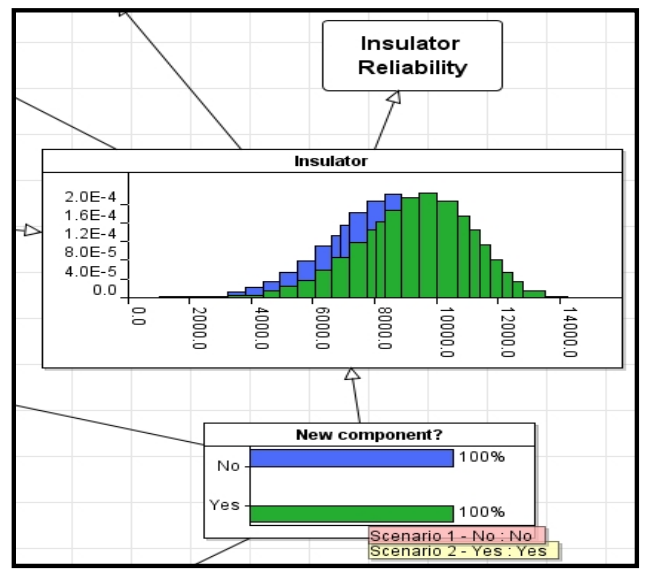

Figure 7. Conditional Reliability 


\section{Conclusions}

It is essential to get more information about the system to make the analysis more efficient; however, based on the available data, the BN analysis shows that the LNG/Propane subsystem is the subsystem that caused the greatest impact on the reliability of the regasification system. Thus, the maintenance plan should pay special attention to this subsystem and consider the inclusion of a redundant subsystem, once that subsystem reliability may increase the regasification system reliability by more than $2 \%$; a redundant subsystem is reasonable and not only the improvement of the critical components of this subsystem (P2 and P3), once they may improve the reliability system by less than $1 \%$. The maintenance plan must pay special attention to the compressor, too, once this is the most critical component of the system given the evidence: system fault. The scenarios analyzed here are only a few examples; many others scenarios can be simulated using the BN capability to model several scenarios, according to the evidences.

The conditional reliability analysis shows that a significant change may occur in the failure probabilities components which do not have exponential distributions. Thus, it is recommendable to perform a detailed study about the failure distribution of each equipment, and then assess the conditional reliability behavior.

It is possible to model different scenarios, such as improvements in critical components, the impact of common cause failures, the impact of including redundancies and any other condition that affects the system reliability.

This chapter described the basic configuration of the regasification system on board a FSRU, evaluated the assessment of its reliability using the proposed methodology allowing to obtain detailed results for the mission time, in addition to permitting to consider some possible scenarios that led to the identification of critical points and considering possible improvements to the system reliability. Besides the evaluation system regasification, this chapter also highlights the potential of BN for the improvement of reliability studies.

In future work, the reliability analysis applied in this study may be expanded to the entire FSRU. In addition, a study about the system dependability may be performed; yet, to perform this study, the assessment of the maintenance process will be necessary. Also, the impact of a failure in this system may reach other areas of the vessel, such as the LNG tanks, which can lead to severe consequences. Therefore, future work may also focus on performing a risk analysis of the FSRU.

\section{Author details}

Marcelo Ramos Martins and Adriana Miralles Schleder

Naval Architecture and Ocean Engineering Department, University of Sao Paulo, Brazil 


\section{References}

[1] Ramos, M. A.; Martins, M. R.; Droguett, E. L.; Souza, E. P. “Quantitative risk analysis and comparison for onshore and offshore LNG terminals: the port of Suape - Brazil Case". In: International Conference on Ocean, Offshore and Arctic Engineering, 2011, Rotterdam, Netherlands. Proceedings of the ASME 2011 30th International Conference on Ocean, Offshore and Arctic Engineering, 2011. p. 1-8.

[2] Natacci, F. B.; Ikeda, N. H.; Martins, M. R. (2010) —Consequence analysis of a liquefied gas leakage\|. Proceedings of the ASME, 29th International Conference on Ocean, Offshore and Artic Enginnering, OMAE 2010.Shanghai, China.

[3] Weber, P., Oliva, G. M., Simon, C., Iung, B., 2010, “Overview on Bayesian Networks applications for dependability, risk analysis and maintenance areas", Engineering Applications of Artificial Intelligence, doi: 10.1016/j.engappai.2010.06.002.

[4] Schleder, A. M. ; Martins, M. R. ; Souza, G. F. M. (2011) —Bayesian networks on risk analysis of a regasification system on an offshore unit.|| In: International Conference on Ocean, Offshore and Arctic Engineering, 2011, Rotterdam, Netherlands. Proceedings of the ASME 2011 30th International Conference on Ocean, Offshore and Arctic Engineering, 2011. p. 1-8.

[5] Agena risk desktop (2011). Available at: http://www.agenarisk.com/ products/desktop.shtml, accessed at 05/04/ 2011.

[6] Neapolitan, R. E. (2004) —Learning Bayesian Networks\|. New Jersey, PearsonPrentice Hall, 2004.

[7] Langseth, H., Portinali, L. (2005) —Bayesian networks in reliabilityll, Reliability Enginnering and System Safety 92, 2007, p.p. 92-108.

[8] Jones. B.; Jenkinson, I.; Yang, Z.; Wang, J. (2009) — The use of Bayesian networking for maintenance planning in a manufacturing industry\|. Reliability Engineering and System Safety 95, 2010, p.p. 267-277.

[9] Marquez, d., Neil, M., Fenton, N. (2010) —Improved reliability modeling using Bayesian Networks and dynamic discretization||. Reliability Enginnering and System Safety 95, 2010, p.p. 412-425.

[10] Marquez, D., Neil, M., Fenton, N. (2011) -A new Bayesian Networks approach to Reliability modeling \|. Available at: http://www.agenarisk.com/studies.shtml, accessed at 05/04/ 2011 .

[11] Bobbio, A.; Portinale, L.; Minichino, M.; Ciancamerla, E. (2001) -Improving the analysis of dependable systems by mapping fault trees into Bayesian networks $\|$. Reliability Enginnering and System Safety 71, 2001, p.p. 249-260.

[12] Netica Software, available at: http://www.norsys.com/, accessed on November, 10th, 2010.

[13] Hugin software, available at: http://www.hugin.com/serene accessed on November, 10th, 2010.

[14] Jensen, F. V., 2001, "Bayesian Networks and decision graphs", Springer, New York, NY.

[15] Schleder, A. M. ; Martins, M. R. ; Souza, G. F. M. (2011) Preliminary risk analysis of a liquefied natural gas regasification system in an offshore unit.\| In: International 
Conference on Vulnerability and Risk Analysis and Management, College Park, Maryland, US. Proceedings of the International Conference on Vulnerability and Risk Analysis and Management, 2011. p. 1-8.

[16] Smith, D. J. (2001) — Reliability, Maintainability and Risk\|. Woburn,UKA. $6^{\underline{a}}$ edição. Butterworth Heinemann.

[17] Schuller, J. C. H (2005) -Methods for determining and processing probabilities - CPR 12E |l. The Hague, Netherlands. $2^{\underline{a}}$ edition. Gervarlijke Stoffen Publicattiereeks.

[18] Charniak, E. (1992) —-Bayesian Networks without tears\|, AI Magazine, 1991,12-4, p.p. 50-63.

[19] Whiiaker, J. (1990) —Graphical Models en apllied multivariate statistics\|, Chichester, UK, Wiley.

[20] OREDA (2002) - Offshore Reliability Data Handbook\|, Det Norske Veritas - DNV, 2002, 4th Edition.

[21] HSE - Health and safety executive (2001) - Offshore Hydrocarbon Releases Statistics, 2001 - Hid Statistics Report HSR 2001 002\|. Available at: www.hse.gov.uk/hid/osd/hsr2001/contents.htm 